\title{
USERS’ PERCEPTIONS OF SOCIAL ISSUES AND THE REPRESENTATION AND INCLINATION TO USE SOCIAL VIDEO (Poster)
}

\begin{abstract}
Associations between users' perceptions of social topics, video representations, and inclinations to use video in social contexts are explored. Online surveys assessed users' preexisting knowledge of a social topic and expectations of a video search, together with post-search impressions and expressed inclinations. Implications complement research on interface evaluation and design.
\end{abstract}

\section{Résumé:}

\section{Research Objective}

The overarching research objective of the current study is to explore associations between users' mental images of topics tied to current social issues and expectations and perceptions of video representations, and, in turn, inclinations to use social video. The current study serves a preliminary examination for better understanding how emerging information and sub-topics of trending social issues correspond to users' expectations, assessment, and decisions to use video information. Immediate insights are provided, including the understanding that video use in social contexts provides additional considerations for both interface design and evaluation.

\section{Method}

An online survey with participatory aspects was employed. "Ferguson, Missouri" was designated as the test topic. "Ferguson" is tied to contemporary social issues and thus is a topic among many social communications and activities across different types of media. The survey included a combination of scaled and open-ended questions (yet, only findings of the scaled questions are reported here).

Prior to a formal search for video on the topic, participants were asked to rate, on a scale of 1-5, their level of:

- Having clear mental visuals when thinking of "Ferguson"

- Having clear expectations of what would be returned from a search for video(s) on the topic

Using a common video search tool, participants were then asked to perform a live search for videos about "Ferguson" and to examine the set of search results. Subsequently, participants were asked to return to the survey and rate post-search responses (again, on a scale of 1-5) including:

- Representations (i.e. surrogates) returned from the search met expectation(s) 
- If searching the topic, I (the user) would feel inclined to select and watch videos from the actual returned set

A total of 330 participatory surveys were collected and analyzed. Descriptive and correlation analyses were performed in order to produce a preliminary set of results as part of the larger research study.

\section{Participants}

Participation was solicited on the campus of a major university, as facilitated through a coordinated subject pool at a research services center. As a result, undergraduate students comprised the majority of the sample, an acceptable limitation of a convenient sample; however, this participant sample fits well within the target audience of the current study, which comprises active users of online video and social media, and thus is suitable for exploring these initial research objectives.

\section{Results}

Quantitative results yielded means with high levels of agreement for each of the variables (as listed above). Mean values ranged from a low of 3.95 to a high of 4.19.

Correlation tests demonstrated statistically significant coefficients for each of the examined relationships. Further, results showed that the level of pre-existing mental images of the experimental topic was positively correlated with users' expectations of the video representations that would be produced from an actual search. Participants' opinions of what would be displayed to them after a search were then positively correlated to their judgments about 1) whether or not the actual displayed representations (post-search) met expectation(s) and 2) the level to which they would feel encouraged to watch video(s) being returned to them. Users' judgments about the actual video representations - in terms of meeting expectations or assumptions - were also positively correlated with the stated levels of likelihood for selecting the video as displayed (through their representations). These results were reasonable, and no unexpected correlations were produced; all coefficients were statistically significant at the $p<0.01$ level.

\section{Discussion and Implications}

It is important that video content be assessed by users in order to determine if a clip is worthy of using or sharing as a basis of interacting with others. The current findings, while exploratory, are significant; results help describe certain phenomena which occur as part of the larger or more holistic interactive search situations involving social video. The current study also complements foundational research on video surrogation (Song \& Marchionini, 2007; Wildemuth, et. al, 2003) by investigating pre-existing expectations of users and testing associations with their perceptions of search and inclinations to use video in a social context. Such results also build upon prior research on users' attitudes for using and adopting interactive video retrieval tools (Albertson \& Ju, 2016; Ju \& Albertson, 2015)

Findings invoke a number of implications. There are implications for design and evaluation which connect traditional perspectives with social connotations surrounding users' assessment, selection, and use of video. Additionally, findings enhance the understanding of video representations beyond user preference metrics of video 
summarizations by showing that facilitating video needs (and thus improving user satisfaction) can be event-driven. One lesson learned from these findings reveals the potential for disconnects of video representations or surrogates in social contexts. For example, new developments or more granular topics pertaining to larger social issues may not be recognizable to or match the existing mental images of users, providing uncertainty (and thus frustration) as part of the video selection and use process.

Therefore, surrogates for social video may be supported by explicating relationships in the context of social issues in order to help users better assess the usefulness of video for their information need. This finding can be supported in different ways including as part of video processing/editing and playback, video surrogation, and/or embedding the video in the context of larger social interactions among users (and groups). These initial implications of the current study will be further tested and reported in future work.

\section{Reference List:}

Albertson, D. \& Ju, B. (2016). Perceived self-efficacy and interactive video retrieval. Journal of Documentation, 72(5).

Ju, B. \& Albertson, D. (2015). Examining user-driven factors for intentions to use video digital libraries. In Proceedings of the Annual Meeting of the Association for Information Science and Technology, November 6-10, St. Louis.

Song, Y., \& Marchionini, G. (2007). Effects of audio and visual surrogates for making sense of digital video. In Proceedings of the SIGCHI Conference on Human Factors in Computing Systems, 867-876.

Wildemuth, B. M., Marchionini, G., Yang, M., Geisler, G., Wilkens, T., Hughes, A., \& Gruss, R. (2003). How fast is too fast?: Evaluating fast forward surrogates for digital video. In Proceedings of the 3rd ACM/IEEE Joint Conference on Digital Libraries, 221-230. 